\title{
Analysis of Solute Distribution in Ice Formed in Progressive Freeze-concentration
}

\author{
Mihiri Gunathilake, Kiyomi Shimmura and Osato MiYawaki \\ Department of Food Science, Ishikawa Prefectural University, 1-308 Suematsu, Nonoichi, Ishikawa 921-8836, Japan
}

Received December 25, 2012; Accepted February 7, 2013

Progressive freeze-concentration of 3 to $30 \%$ sucrose solutions were carried out using a small cylindrical apparatus. The solute concentration distribution in the ice phase was determined by cutting the ice formed into fractions and analyzing the solute concentration in the fractions. The solute concentration in the ice phase was found to increase as the concentration process progressed. From the solid phase analysis, the corresponding liquid phase concentration was estimated in the concentration process and an effective partition coefficient, $K$, was determined. $K$ was also obtained from the concentration polarization theory. To this purpose, the limiting partition coefficient, $K_{0}$, was obtained, then $K$ was determined from the concentration polarization theory. $K$ obtained by the two methods agreed well. $K$ increased with an increase in solute concentration of sucrose, which corresponds to the higher incorporation rate of solute into the ice phase in progressive freeze-concentration.

Keywords: progressive freeze-concentration, solute distribution in ice phase, effective partition coefficient, limiting partition coefficient

\section{Introduction}

High quality concentration of liquid food is an important unit operation in the food industry. With increasing consumer demands for liquid foods with natural taste, aroma, nutrients, and colors, the concentration of liquid food without deteriorating the original quality of the food is a challenge. Due to the low process temperature and completely selective dewatering, freeze-concentration is the best method in terms of retaining the original quality of the liquid food among the other methods for liquid food concentration (Deshpande et al., 1982).

The commercial freeze-concentration method currently available is known as suspension crystallization. In this method, small ice crystals formed in a heat-exchanger-crystallizer are transferred to a recrystallizer, where ice crystals are allowed to grow by Ostwald ripening mechanism (Huige and Thijssen, 1972). The ice crystals are then transferred to a washing column to separate the ice crystals from the mother solution. This system has extensively been investigated due to its adaptability to scale-up; however, the complexity of the system results in high capital costs and limited practical application of this method.

*To whom correspondence should be addressed.

E-mail: osato@ishikawa-pu.ac.jp
Progressive freeze-concentration is a new freeze concentration method, where a single ice crystal is formed by a moving ice front from one end of the sample vessel to the other end (Liu et al., 1997). Therefore, it is easy to separate the ice crystal from the concentrated solution. A falling film reactor has been developed for the large scale production with progressive freeze-concentration (Flesland, 1995) and has been used to concentrate apple and pear juices (Hernandez et al., 2009), orange juice (Sanchez et al., 2010), grape must (Hernandez et al., 2010) and whey (Sanchez et al., 2011). The effective separation of solute from the ice phase requires a high mass transfer at the ice-liquid interface; thus high flow rate is an important operational parameter for the efficiency in progressive freeze-concentration. Due to the limited flow rate, however, the mass transfer at the ice-liquid interface is limited in the falling film reactor. In addition, the falling film reactor has a free open-air surface, resulting in loss of volatile flavor components.

A closed tubular ice system with circulating flow was developed to increase the mass transfer and productivity of the progressive freeze-concentration (Miyawaki et al., 2005). In this system, the ice crystal grows on the inside surface of a pipe cooled by a coolant. This tubular system can be easily scaled up by increasing the surface area of the cooling plate. Coffee extract, tomato juice and sucrose solutions have been 
successfully concentrated using this system, providing highly concentrated liquid (Miyawaki et al., 2005). In progressive freeze-concentration, yield decreases with an increase in solute concentration of the sample because of the higher incorporation rate of solute into the ice phase. To counteract this, partial melting of the ice has been proposed to improve the yield (Miyawaki et al., 2012). Coupling of the partial melting technique with its simple processing system, progressive freeze-concentration is expected as a promising technique for the high quality concentration of liquid food.

The effective partition coefficient is practically an important parameter which determines the solute separation efficiency in progressive freeze-concentration. The present paper analyzes, for the first time, the concentration distribution of a solute in the solid-ice phase by cutting the ice formed in the progressive freeze-concentration of sucrose solutions into fractions, allowing the effective partition coefficient to be obtained from the solute distribution in ice. The effective partition coefficient is also obtained using the concentration polarization theory (Miyawaki et al., 1998). The effective partition coefficient values obtained by the two methods are compared.

\section{Theoretical Considerations}

Effective partition coefficient in progressive freezeconcentration The effective partition coefficient of solute between the ice and liquid phase in progressive freezeconcentration is defined by the following equation (Liu et al., 1997).

$$
K=C_{S} / C_{L}
$$

where, $C_{S}$ and $C_{L}$ are the solute concentrations in the ice and liquid phases, respectively. The mass balance of the solute in the ice and liquid phases at the moment, when the solution volume is $V_{L}$ in the process of progressive freeze-concentration, is described as follows (Liu et al., 1997).

$$
C_{L} V_{L}=-C_{S} d V_{L}+\left(C_{L}+d C_{L}\right)\left(V_{L}+d V_{L}\right)
$$

From Eqs. (1) and (2), the following differential equation is obtained.

$$
\left(d C_{L} / C_{L}\right) /\left(d V_{L} / V_{L}\right)=K-1
$$

If the effective partition coefficient, $K$, is assumed to be constant throughout the concentration process, Eq. (3) can be integrated as follows.

$$
(1-K) \ln \left(V_{L} / V_{0}\right)=\ln \left(C_{0} / C_{L}\right)
$$

where $V_{0}$ and $C_{0}$, respectively, are the initial volume of solution to be concentrated and the initial solute concentration in the liquid phase. This equation can be rearranged as follows:

$$
C_{L} / C_{0}=\left(V_{0} / V_{L}\right)^{1-K}
$$

According to this, from the ratios of $C_{L} / C_{0}$ and $V_{0} / V_{L}$, the effective partition coefficient $K$, can be obtained.

Concentration polarization model and limiting partition coefficient The effective partition coefficient has been theoretically described by the concentration polarization theory (Miyawaki et al., 1998) as follows.

$$
K=K_{0} /\left[K_{0}+\left(1-K_{0}\right) \exp (-u / k)\right]
$$

where $K_{0}$ is the limiting partition coefficient, $u$ is the advance rate of ice front, and $k$ is the mass transfer coefficient at the ice-liquid interface, which has been related to the stirring rate, $N$, as follows (Miyawaki et al., 1998).

$$
k=a N^{0.2}
$$

where $a$ is a parameter experimentally determined. The limiting partition coefficient is defined as the partition coefficient at the ice-liquid interface.

$$
K_{0}=C_{S} / C_{i}
$$

where $C_{S}$ and $C_{i}$, respectively, are the solute concentrations in the ice and solution phases at the very interface. Although $C_{i}$ can be clearly defined theoretically, it is difficult to measure experimentally. Alternatively, the limiting partition coefficient, $K_{0}$, is considered to be the partition coefficient at an infinitesimal ice crystal growth rate and/or infinite mass transfer coefficient in Eq. (6). In consideration of Eq. (7), Eq. (6) can be rearranged as follows (Pradistsuwana et al., 2003).

$$
\ln (1 / K-1)=\ln \left(1 / K_{0}-1\right)-(1 / a)\left(u / N^{0.2}\right)
$$

From this, a linear dependence of $\ln (1 / K-1)$ on $u / N^{0.2}$ is expected, and $K_{0}$ can be obtained from the extrapolation of the linear plot to $u / N^{0.2} \rightarrow 0$ (Gu et al., 2005).

\section{Materials and Methods}

Apparatus for progressive freeze-concentration A small cylindrical test apparatus (Fig. 1) was used for progressive freeze-concentration ( $\mathrm{Gu}$ et al., 2005). Two types of cylindrical sample vessels were used in this study. One vessel consisted of a plastic side with a stainless steel bottom (49 $\mathrm{mm}$ diameter, $168 \mathrm{~mm}$ height) and the other one was a fully stainless steel vessel (47 $\mathrm{mm}$ diameter, $198 \mathrm{~mm}$ height). The sample vessel was plunged into a cooling bath (BB 400, Yamato Scientific, Tokyo, Japan) at a constant speed. The advance rate of the ice front was calculated from the thickness of the ice formed and the duration of the experiment. The sample vessel was equipped with a stirrer (SM-102, As One, Osaka, Japan) and a 4-blade propeller (4 cm in diameter) for stirring the solution at the ice-liquid interface. 


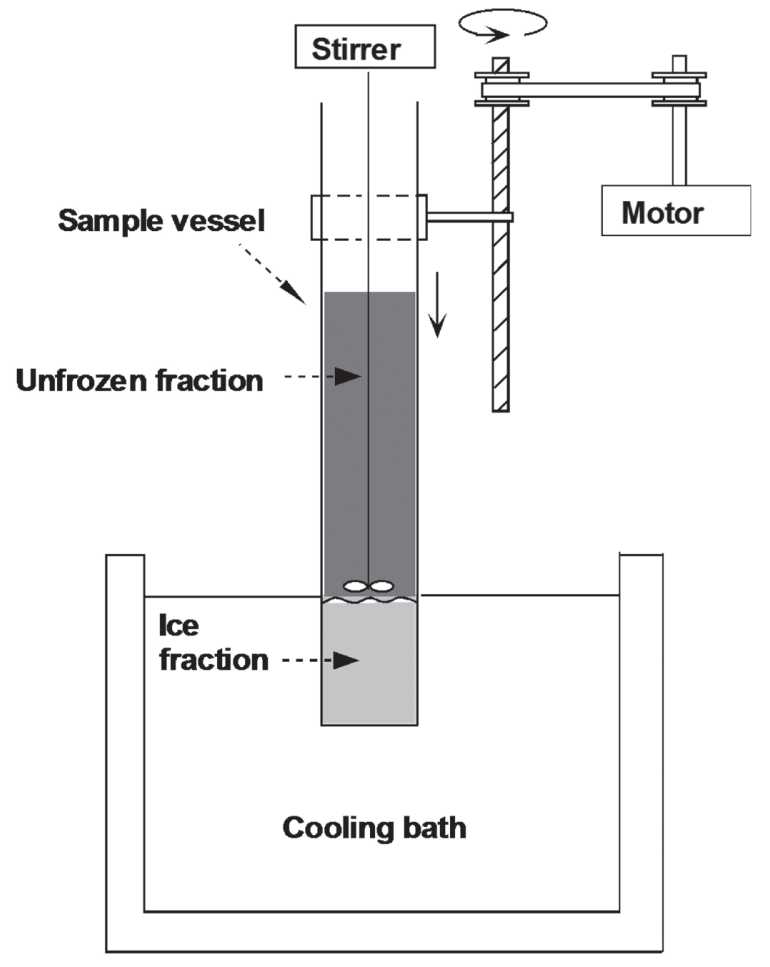

Fig. 1. A small cylindrical test apparatus for progressive freezeconcentration.

Sucrose solutions of various concentrations were used as samples to be concentrated. Sucrose was obtained from Kanto Chemicals (Tokyo, Japan). The concentration of the sucrose solutions was determined using a refractometer (APAL-1, As One, Osaka, Japan).

Determination of effective partition coefficient from solute distribution in ice-phase in progressive freeze-concentration Sucrose solutions with concentrations of $3 \mathrm{wt} \%, 10 \mathrm{wt} \%, 20$ $\mathrm{wt} \%$ and $30 \mathrm{wt} \%$ were progressive freeze-concentrated using the stainless steel vessel. Pure water of $1 \mathrm{~mL}$ was added to the bottom of the vessel to provide a seed ice crystal to avoid initial supercooling (Liu et al., 1997). The sample volume was $150 \mathrm{~mL}$ in all experiments. A fully stainless steel vessel was used because effective heat transfer from the side surface is necessary to ensure a constant ice crystal growth rate during long time operation. Before being added to the vessel, the sample solution was precooled to the freezing point to prevent melting of the seed ice crystal. All experiments were carried out with a stirring speed of $1000 \mathrm{rpm}$ at the ice-liquid interface and a moving speed of ice front at $1 \mathrm{~cm} / \mathrm{h}$ for $5 \mathrm{~h}$. The cooling bath temperature was $-20^{\circ} \mathrm{C}$ except for the $3 \%$ solution sample, for which the cooling bath temperature was $-15^{\circ} \mathrm{C}$. After the freeze-concentration experiment, the single ice crystal formed was separated from the concentrated solution and was cut by a saw into three fractions of roughly equal amount. The volume and the concentration of the solute in the three ice fractions and in the concentrated solution were measured after melting the ice.

Effective partition coefficient based on concentration polarization model Sucrose solutions of 5, 15, and $25 \mathrm{wt} \%$ were progressive freeze-concentrated at various operating conditions using the plastic sample vessel. In this vessel, the heat transfer from the side surface is negligible, so a strict uni-dimensional ice crystal growth was expected. The stirring rate at the ice-liquid interface was varied from $300 \mathrm{rpm}$ to $1300 \mathrm{rpm}$. The moving speed of ice front was varied from $0.35 \mathrm{~cm} / \mathrm{h}$ to $2.0 \mathrm{~cm} / \mathrm{h}$. The cooling bath temperature was set at $-15^{\circ} \mathrm{C}$ for all the experiments. For the lowest moving speed of ice front at $0.35 \mathrm{~cm} / \mathrm{h}$, the experiments were repeated also with the cooling bath temperature at $-10^{\circ} \mathrm{C}$. Before starting the freeze concentration experiment, $1 \mathrm{~mL}$ of pure water was added to the bottom of the vessel to provide seed ice crystal for preventing initial supercooling (Liu et al., 1997). The sample solution of $150 \mathrm{~mL}$ was precooled to the freezing point in order to avoid melting of the seed ice crystal before its transfer into the sample vessel. The concentration process was continued until an approximately $1 \mathrm{~cm}$ thick ice crystal was formed in the sample vessel, then the concentrated solution was separated and the ice layer was allowed to melt. The volume and the sucrose concentration of the melted ice and the concentrated solution were measured and the exact thickness of the ice crystal was determined from the volume of the melted ice.

\section{Results and Discussion}

Progressive freeze-concentration of sucrose solution and analysis of solute distribution in ice phase Progressive freeze-concentration of various sucrose solutions was carried out with the same operating conditions of ice crystal growth rate at $1 \mathrm{~cm} / \mathrm{h}$ and stirring rate at $1000 \mathrm{rpm}$.

Table 1 summarizes the results. In the freeze concentration process with volumetric concentration ratios, $V_{0} / V_{L}$, between 2.5 to 3.5 , concentration ratios, $C_{L} / C_{0}$, of 1.5 to 2.9 were obtained. In this table, total volume of ice formed and concentrated liquid is little lower than that of initial solution mainly because of the loss in the ice cutting process. When the initial sucrose concentration was $3 \%$, a concentrated solution of $8.7 \%$ was obtained with a yield of $82.2 \%$. With an increase in solute concentration, the yield decreased to $58.6 \%$ for the $30 \%$ sucrose solution, although the final concentration for this solution was as high as $45 \%$. This lower yield for the higher concentrated sample is a major drawback of progressive freeze-concentration. However, the partial melting-ice technique has been reported to be effective to improve the yield for highly concentrated solutions (Miyawaki et al., 2012).

Table 2 shows how the solute concentration varies in the 
Table 1. Progressive freeze-concentration of sucrose solutions.

\begin{tabular}{|c|c|c|c|c|c|c|c|}
\hline $\begin{array}{c}\text { Initial solute } \\
\text { conc. } \\
C_{0}\left(\mathrm{wt}^{0} \%\right)\end{array}$ & $\begin{array}{c}\text { Final volume } \\
V_{L}(\mathrm{~mL})\end{array}$ & $\begin{array}{l}\text { Volume ratio } \\
\qquad V_{0} / V_{L}\end{array}$ & $\begin{array}{l}\text { Final liq. conc. } \\
C_{L}(\mathrm{wt} \%)\end{array}$ & $\begin{array}{l}\text { Conc. ratio } \\
\qquad C_{0} / C_{L}\end{array}$ & $\begin{array}{l}\text { Total ice volume } \\
\qquad V_{S}(\mathrm{~mL})\end{array}$ & $\begin{array}{l}\text { Conc. in } \\
\text { ice phase } \\
C_{S}\left(\mathrm{wt}^{0} \%\right)\end{array}$ & $\begin{array}{l}\text { Yield } \\
(\%)\end{array}$ \\
\hline 3 & 42.5 & 3.53 & 8.7 & 2.9 & 99.2 & 0.745 & 82.2 \\
\hline 10 & 54.5 & 2.75 & 20.2 & 2.02 & 89.0 & 3.66 & 73.4 \\
\hline 20 & 61 & 2.46 & 32.2 & 1.61 & 85.2 & 10.82 & 65.5 \\
\hline 30 & 59 & 2.54 & 44.7 & 1.49 & 88.8 & 18.93 & 58.6 \\
\hline
\end{tabular}

*) Initial volume of solution before freeze concentration, $V_{0}$, was kept constant at $150 \mathrm{~mL}$.

Table 2. Concentration distribution of solute in solid phase in progressive freeze-concentration of sucrose solutions.

\begin{tabular}{cclcc}
\hline$C_{0}(\mathrm{wt} \%)$ & $V_{S}(\mathrm{~mL})$ & Ice location & $\begin{array}{c}\text { Volume of ice } \\
\text { fraction }(\mathrm{mL})\end{array}$ & $C_{S}(\mathrm{wt} \%)$ \\
\hline \multirow{2}{*}{3} & \multirow{2}{*}{99.15} & Bottom & 34.5 & 0.31 \\
& & Middle & 38.4 & 0.62 \\
& & Top & 26.3 & 1.24 \\
\multirow{2}{*}{10} & \multirow{2}{*}{88.95} & Bottom & 37.9 & 2.83 \\
& & Middle & 27.1 & 3.25 \\
& & Top & 24.0 & 5.45 \\
\multirow{2}{*}{20} & \multirow{2}{*}{85.2} & Bottom & 34.1 & 8.39 \\
& & Middle & 28.2 & 10.79 \\
& & Top & 22.9 & 14.48 \\
30 & \multirow{3}{*}{88.8} & Bottom & 25.8 & 15.85 \\
& & Middle & 31.8 & 17.47 \\
& & Top & 31.2 & 22.96 \\
\hline
\end{tabular}

ice phase during the concentration process. The bottom fraction of the ice represents the initial ice fraction formed at the beginning of the concentration process, the middle fraction represents the middle stage of the concentration and the top ice fraction represents the last stage of concentration. With the progress in the concentration process, the solute concentration in the liquid phase increases from the bottom to the top, and the amount of solute incorporated into the solid ice phase increases gradually. This causes a higher solute concentration in the top ice fraction compared with the bottom fraction.

From the concentration and the volume of each ice fraction, the solute concentration in the solution phase, $C_{L}$, at the end of ice crystal growth of each ice fraction can be calculated by the mass balance equation. Thus $C_{L} / C_{0}$ was obtained and compared with $V_{0} / V_{L}$ according to Eq. (5). Figure 2 shows the results. Straight lines are obtained in the log-log plot between $C_{L} / C_{0}$ and $V_{0} / V_{L}$. This was rather unexpected because the effective partition constant, $K$, was supposed to increase experimentally with the concentration process although a constancy was assumed in the derivation of Eq. (5). The limiting partition coefficient, $K_{0}$, is solute-concentration dependent (Gu et al., 2005) so that $K$ is also concentration- dependent according to the concentration polarization theory (Miyawaki et al., 1998). Therefore, the plot in Fig. 2 was expected to be a curved line but straight lines were obtained for each condition. From the slope of the plot in Fig. 2, $K$ values were obtained to be $0.150,0.270,0.437,0.521$ for $3 \mathrm{wt} \%, 10$ $\mathrm{wt} \%, 20 \mathrm{wt} \%, 30 \mathrm{wt} \%$ sucrose solutions, respectively.

Limiting and effective partition coefficient by concentration polarization model The effective partition coefficient, $K$, was experimentally measured for $5 \mathrm{wt} \%, 15 \mathrm{wt} \%$ and $25 \mathrm{wt} \%$ sucrose solutions by forming a small amount of ice crystal for different combinations of ice growth rates, $u$ and stirring rates, $N$. From Eq.(9), a linear relationship is expected between $u / N^{0.2}$ and $\ln (1 / K-1)$. Figure 3 shows the results for a $5 \%$ sucrose solution. Although data are little scattering mainly because of the difficulty in controlling the clearance between the stirrer and the ice surface, a linear line was obtained statistically. Extrapolation of this linear line to $u / N^{0.2} \rightarrow 0$ allowed the limiting partition coefficient, $K_{0}$, to be obtained. Thus obtained $K_{0}$ values were $0.131,0.227$, 0.363 , respectively, for $5 \mathrm{wt} \%, 15 \mathrm{wt} \%$, and $25 \mathrm{wt} \%$ sucrose solutions (Table 3 ). In a previous study, $K_{0}$ has been obtained

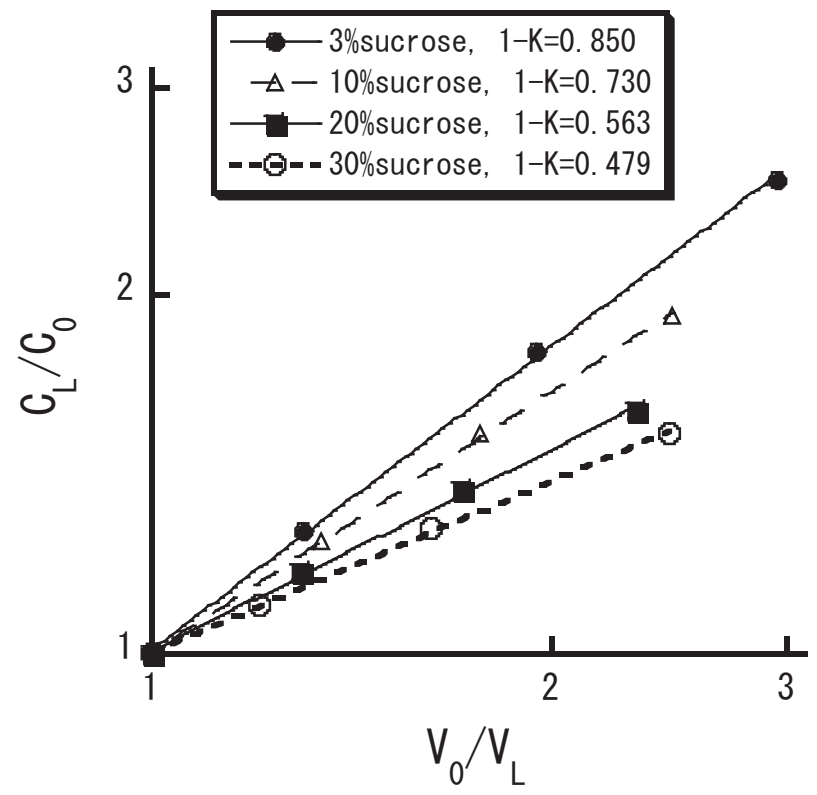

Fig. 2. Concentration process in progressive freeze-concentration of sucrose solutions as determined by solid phase analysis. 


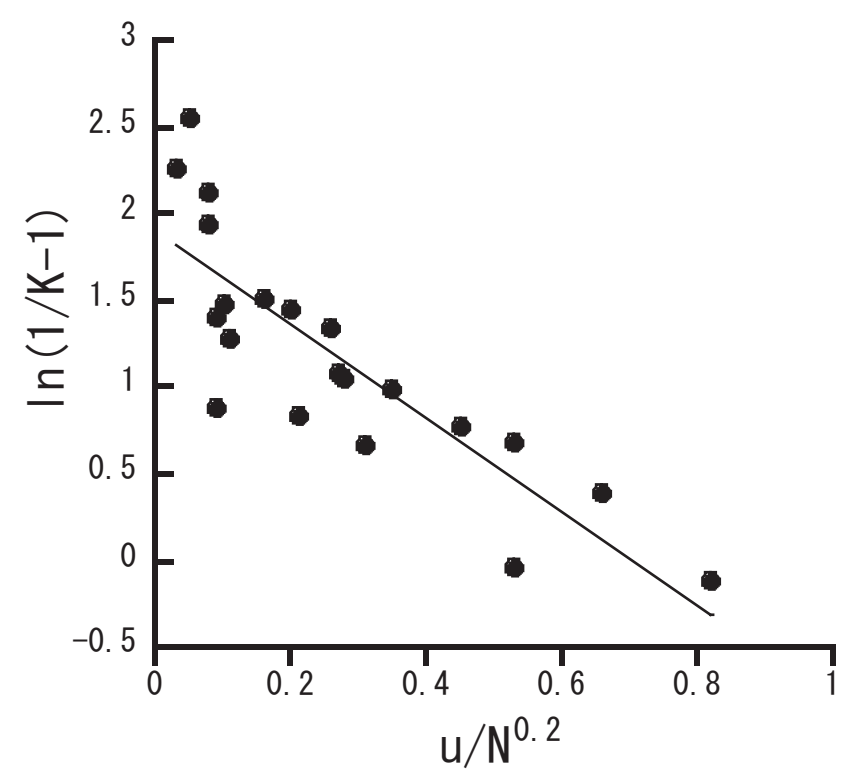

Fig. 3. Determination of limiting partition coefficient in progressive freeze-concentration for $5 \%$ sucrose solution.

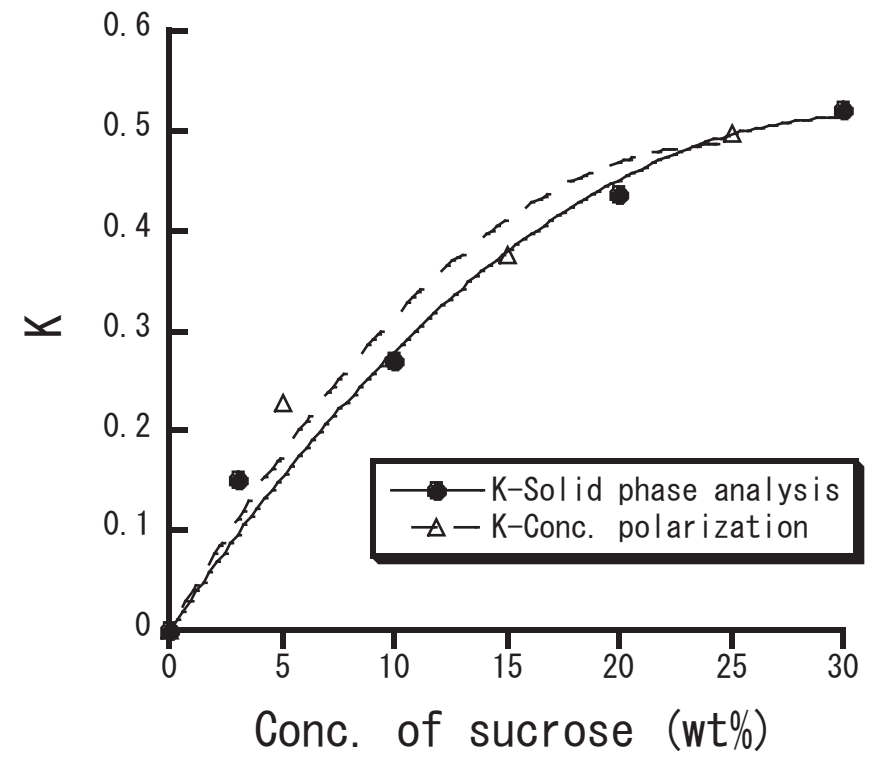

Fig. 4. Comparison of effective partition coefficients determined from ice phase analysis and concentration polarization theory in progressive freeze-concentration of sucrose solution.

Table 3. Limiting partition coefficient for sucrose in progressive freeze-concentration.

\begin{tabular}{ccccccc}
\hline $\begin{array}{c}\text { Sucrose conc. } \\
(\text { wt } \%)\end{array}$ & $\begin{array}{c}\text { Limiting Partition } \\
\text { Coeff. }\left(K_{0}\right)\end{array}$ & $\begin{array}{c}\text { Parameter } a \\
\text { in Eq. }(7)\end{array}$ & $\begin{array}{c}\text { Correlation } \\
\text { coefficient }\end{array}$ & $\begin{array}{c}\text { Effective partition } \\
\text { coeff. }(K)\end{array}$ & $\begin{array}{c}\text { Osmotic pressure } \\
(\mathrm{MPa})\end{array}$ & $K_{0}$ for salts $\left.{ }^{*}\right)$ \\
\hline $5 \%$ & 0.131 & 0.372 & 0.845 & 0.228 & 0.388 & 0.02 \\
$15 \%$ & 0.227 & 0.350 & 0.812 & 0.376 & 1.36 & 0.09 \\
$25 \%$ & 0.363 & 0.457 & 0.695 & 0.497 & 2.69 & 0.16 \\
\hline
\end{tabular}

${ }^{*}$ Estimated from Gu et al. (2005).

for various salt solutions and was found to correlate well to the osmotic pressure of the solutions (Gu et al., 2005). In Table 3, the osmotic pressure is shown so that $K_{0}$ values obtained here are compared with the literature value for salts. Then it turned out that $K_{0}$ values obtained for sucrose are much higher than those for salts. There are many differences between salts and sugars in molecular structure, size, and charge, which might have caused the difference in the affinity between solute and ice. In the literature, the difference in $K_{0}$ between salts and glucose has been discussed (Gu et al., 2005).

From the limiting partition coefficient, $K_{0}$, obtained, the effective partition coefficient, $K$, was calculated by using Eqs. (6) and (7). As shown in Table 3, both $K$ and $K_{0}$ increased with an increase in solute concentration. However, there was a difference between $K$ and $K_{0}$ with the former being much larger than the latter. This reflects the limiting effect of the operating conditions of the advance rate of ice front and the stirring rate on $K$.

Comparison of effective partition coefficients obtained from ice-phase analysis and concentration polarization model The effective partition coefficient, $K$, is practically very important because this parameter determines the amount of solute to be incorporated into the ice phase, which directly affects the yield in progressive freeze-concentration. The $K$ values obtained from the ice phase analysis and the concentration polarization theory are compared in Fig. 4 showing a good agreement between the two. The $K$ from the ice phase analysis is an average value in the freeze-concentration process so that this was expected to be higher than that from the concentration polarization theory in principle. In practice, however, a good agreement was observed between the two.

\section{Conclusions}

Solute distribution analysis of the ice phase was effectively carried out, for the first time, in the progressive freezeconcentration of sucrose solutions with concentration varied from $3 \mathrm{wt} \%$ to $30 \mathrm{wt} \%$. The solute concentration in the ice phase was shown to increase with the progress in the concentration process. From the solute concentration in the solid phase, the corresponding liquid phase concentration in the concentration process was calculated. From this, an effective partition coefficient, $K$, was obtained for progressive freezeconcentration. 
$K$ was also obtained from the concentration polarization model. To this purpose, the limiting partition coefficient, $K_{0}$, was obtained by measuring $K$ at various operating conditions of the advance rate of ice front and the stirring rate. The $K_{0}$ obtained for sucrose was much higher than those for salts in the literature. $K$ was determined from concentration polarization theory. Thus obtained $K$ agreed well with that obtained from the ice phase analysis. $K$ values from the two methods increased with an increase in solute concentration of sucrose, which corresponds to the higher incorporation rate of solute into the ice phase in progressive freeze-concentration.

Acknowledgement Technical help by Ms. Michiko Dozen is highly appreciated.

\section{References}

Deshpande, S.S., Bolin, H.R. and Salunkhe, D.K. (1982). Freeze concentration of fruit juices. Food Technol., May, 68-82.

Flesland, O. (1995). Freeze-concentration by layer crystallization. Drying Technol., 13, 1713-1739.

Gu, X., Suzuki, T. and Miyawaki, O. (2005). Limiting partition coefficient in progressive freeze-concentration. J. Food Sci., 70(9), 546-551.

Hernandez, E., Raventos, M., Auleda, J.M. and Ibarz, A. (2009). Concentration of apple and pear juices in a multi-plate freeze concentrator. Innovat. Food Sci. Emerg. Technol., 10, 348-355.

Hernandez, E., Raventos, M., Auleda, J.M. and Ibarz, A. (2010).
Freeze concentration of must in a pilot plant falling film cryoconcentrator. Innovat. Food Sci. Emerg. Technol., 11, 130-136.

Huige, N.J.J. and Thijssen, H.A.C. (1972). Production of large crystals by continuous ripening in a stirrer tank. J. Crystal Growth, 13/14, 483-487.

Liu, L., Miyawaki, O. and Nakamura, K (1997). Progressive freezeconcentration of model liquid food, Food Sci. Technol. Int. Tokyo, 3(4), 348-352.

Miyawaki, O., Liu, L. and Nakamura, K. (1998). Effective partition constant of solute between ice and liquid phases in progressive freeze-concentration. J. Food Sci., 63(5), 756-758.

Miyawaki, O., Liu, L., Shirai, Y., Sakashita, S. and Kagitani, K. (2005). Tubular ice system for scale-up of progressive freezeconcentration. J. Food Eng., 69, 107-113.

Miyawaki, O., Kato, S. and Watabe, K. (2012). Yield improvement in progressive freeze-concentration by partial melting of ice. $J$. Food Eng., 108, 377-382

Pradistsuwana, C., Theprugsa, P. and Miyawaki, O. (2003). Measurement of limiting partition coefficient in progressive freezeconcentration. Food Sci. Technol. Res., 9(2), 190-192.

Sanchez, J., Ruiz, Y., Raventos, M., Auleda, J.M. and Hernandez, E. (2010). Progressive freeze concentration of orange juice in a pilot plant falling film. Innovat. Food Sci. Emerg. Technol., 11, 644651.

Sanchez, J., Hernandez, E., Auleda, J.M. and Raventos, M. (2011) Freeze concentration of whey in a falling-film based pilot plant: Process and characterization. J. Food Eng., 103, 147-155. 